\title{
Older adult patients at the Emergency Department
}

Citation for published version (APA):

Wachelder, J. J. H. (2021). Older adult patients at the Emergency Department. [Doctoral Thesis, Maastricht University]. Ridderprint. https://doi.org/10.26481/dis.20211130jw

Document status and date:

Published: 01/01/2021

DOI:

10.26481/dis.20211130jw

Document Version:

Publisher's PDF, also known as Version of record

\section{Please check the document version of this publication:}

- A submitted manuscript is the version of the article upon submission and before peer-review. There can be important differences between the submitted version and the official published version of record.

People interested in the research are advised to contact the author for the final version of the publication, or visit the DOI to the publisher's website.

- The final author version and the galley proof are versions of the publication after peer review.

- The final published version features the final layout of the paper including the volume, issue and page numbers.

Link to publication

\footnotetext{
General rights rights.

- You may freely distribute the URL identifying the publication in the public portal. please follow below link for the End User Agreement:

www.umlib.nl/taverne-license

Take down policy

If you believe that this document breaches copyright please contact us at:

repository@maastrichtuniversity.nl

providing details and we will investigate your claim.
}

Copyright and moral rights for the publications made accessible in the public portal are retained by the authors and/or other copyright owners and it is a condition of accessing publications that users recognise and abide by the legal requirements associated with these

- Users may download and print one copy of any publication from the public portal for the purpose of private study or research.

- You may not further distribute the material or use it for any profit-making activity or commercial gain

If the publication is distributed under the terms of Article $25 \mathrm{fa}$ of the Dutch Copyright Act, indicated by the "Taverne" license above, 
CHAPTER 10

Summary 
This chapter will give a brief summary of this thesis.

In chapter 2, we provided a detailed overview of the organisation of the Dutch ED care. We focus on the specialty of acute internal medicine and on the presence and roles of emergency physicians (EP). This overview (based on a questionnaire) may be of additional value regarding an overview of the Dutch ED care. Older adult ED patients are mainly assessed by three specialities: EPs, internists and geriatricians. These specialities may differ in organisation (such as physical presence at the ED, presence of acute internal specialist, working hours) and clinical reasoning, especially concerning the complex older adult patients. It is already well know that physicians will acknowledge that the care of older ED patients poses questions, sets challenges and also may lead to frustration. The overview of the ED organization (such as presence of an EP) with focus on the role of internists gives insight in local solutions for the organization of acute care at the ED. We found that in the emergency care in the Netherlands are many differences in the presence of (acute) internists and EPs in the ED and in working and supervision agreements.

Our study showed that between January 2013 and December 2017, the total number of patients visiting the ED decreased. In contrast, we showed an increase in the number of older adult patients in the same time frame (we only found a slightly lower rate in 2016 as there were less ED visits by older adult patients). Additionally, we identified differences in the presence of (acute) internists and EPs in the ED and in working and supervision agreements. Internists reported their roles at the ED most often as practitioner and consultant. Internists experienced the collaboration with EPs as satisfactory. Furthermore, we found that internists and EPs differ in their way of organisation (such as ED staffing and working hours) and way of working at the ED. It seems that there is no uniform interpretation of how acute care should be organised. Probably the local differences in the demand of care can be a main driver, together with the financial situation, the strategical view of the hospital and preferences of the medical specialists.

In Chapter $\mathbf{3}$ and $\mathbf{4}$, potential risk factors were assessed. We investigated if factors already found associated with poor prognosis in older adult patients add additional risk when presenting to the ED. Two potential risk factors were investigated: weekend admissions and socioeconomic status (SES) of older adult patients presenting to the ED. The weekend effect is defined as the association between weekend admissions, as compared to weekday admissions, and their adverse outcomes.

In chapter 3, we found that emergency care for the older adult ED patients during weekends is not compromised. In our retrospective study, we did not find a weekend effect in older adult internal medicine patients. There was no difference in 2-day and 30-day mortality rate between those admitted during weekends and weekdays. This may 
be surprising, because we found a higher comorbidity and urgency level in older adult patients hospitalised during weekends than during weekdays.

In Chapter 4, we found that having a low Socioeconomic status (SES) is associated with a higher risk of hospitalisation in older adult ED patients than having a high SES. SES categories were based on average household income at zip code level; low (<€1800/ month), intermediate (€1800-€2300/month) and high (>€2300/month). In a large retrospective study, we investigated the association of SES with ED visits. We found that older adult community-dwelling ED patients with a low SES have a higher risk of hospitalisation than patients with a high SES. Additionally, low SES patients more often had a higher triage level, had more diagnostics tests and a longer ED length-of-stay compared with higher SES groups. However, we could not found a differences between in-hospital mortality and the number of ED return visits between the SES groups.

In Chapter 5-7, we evaluated older adult patients presenting with nonspecific complaints (NSC) at the ED. There is a major lack of knowledge on these patients, as they have not been these studied intensively in the literature. The next chapters are based on different definitions concerning NSC. In chapter 5, a retrospective design study, we found that older ED patients with NSC have more comorbidity and polypharmacy than those with specific complaints (SC). In addition, older adult patients presenting with NSC are at higher risk of hospitalization and 30-day mortality than patients presenting with SC.

We provided a descriptive overview of the characteristics and the adverse outcomes of 244 older adult patients who presented as NSC at the ED and compared these with those of SC patients. NSC was defined based on the main referral reason NSC which was noted in the patient electronic ED record. The prevalence of NSC among all older adult patients was $13.7 \%$. These patients with NSC were more often hospitalized and had longer a hospital length of stay than patients with SC. The most important finding was that patients with NSC had a higher 30-day mortality rate compared to patients presenting with SC. The number of ED-return visits within 90 days was the only adverse outcome that was not different between the two groups. This study showed that NSC are associated with adverse outcome.

In Chapter 6, in a retrospective design study, we selected all patients (young and old) presenting as having NSC using an algorithm that includes items that can be obtained early in the ED process. This algorithm was based on the BANC study (1). The selected patients with NSC were mostly older with more comorbidity and polypharmacy than those with SC. In comparison with chapter $\mathbf{5}$ the selected population was smaller compared (13.7\% vs $4.2 \%)$. This will probably be due to the study design, as we examined in the first study the referral reason - as noted by the resident - and in the second study, we thoroughly examined the anamnesis and applied an extensive criteria set, which we thought is extremely important in the diagnosis of patients. 
We showed that NSC patients again had a longer ED length of stay, were more often hospitalized, had a longer hospital length of stay and a substantially higher (11.8 vs. $3.5 \%)$ in-hospital mortality in comparison with SC patients. The similar results between different ways in composing the NSC populations is interestingly. Our study shows that it is feasible to identify NSC patients using an algorithm early in the work-up in the ED and that these identified NSC patients have a poor prognosis.

In Chapter 7, we found that compared to NSC and SC patients readmission were not better predictable nor preventable. We investigated, in a retrospective design, whether patients with NSC differed regarding their readmissions and whether they were more predictable and preventable using the CURIOSA database (2). The CURIOSA study was a prospective, observational study in which readmitted patients, their carers, and treating professionals were interviewed during their readmission to assess the discharge process and the predictability and preventability of the readmission.

We did not find any significant differences between the patients who were readmitted and presented during their first admission with NSC and SC, except that patients with NSC had more comorbidities. Approximately one third of patients felt their readmission was predictable (i.e. $31.0 \%$ of those with NSC versus $27.5 \%$ of those with SC). However, patients with NSC were less likely to find their readmissions preventable than patients with SC (14.9 vs. $25.4 \%)$. Readmissions among those with NSC (11.5\%) were not found to be more often potentially preventable than those with SC (14.9\%) by all who were interviewed.

In Chapter 8, we showed that telemonitoring may be an interesting target for intervention among older adults patients in their home situation. However, the current technology needs practical alterations to serve as suitable intervention to monitor older adult patients in their home situation. It is largely dependent on technology acceptance by older adults if implementation telemonitoring, defined as the collection of symptoms, vital and functional parameters for chronic conditions at home, will be successful. 


\section{References}

1. Nemec M, Koller MT, Nickel CH, Maile S, Winterhalder C, Karrer C, et al. Patients presenting to the emergency department with non-specific complaints: the Basel Non-specific Complaints (BANC) study. Acad Emerg Med 2010 Mar;17(3):284-292.

2. van Galen LS, Brabrand M, Cooksley T, van de Ven PM, Merten H, So RK, et al. Patients' and providers' perceptions of the preventability of hospital readmission: a prospective, observational study in four European countries. BMJ Qual Saf 2017 Jun 22. 


$$
97
$$


CHAPTER 11

Dutch Summary 


\section{Introductie}

Het aantal oudere in de populatie neemt wereldwijd toe. In Nederland is $19,5 \%$ van de populatie 65 jaar of ouder; hiervan is $25 \% 80$ jaar of ouder (1). De gemiddelde levensverwachting voor mannen is 80,4 en voor vrouwen 83,6 jaar (2). De spoedeisende hulp (SEH) is een organisatie welke 24 uur per week zorgt levert voor de patiënten in Nederland. Ouderen patiënten bezoeken de SEH vaker dan jongvolwassenen (3-5), $12-29 \%$ van de SEH bezoeken zijn door deze oudere patiënten. De verwachting is dat het aantal zal verdubbelen in de komende jaren (3-5).

Oudere patiënten hebben vaker, maar ook complexe zorg nodig. Ze hebben een hogere kans op een delier, vallen, infecties en medicatie fouten (4,6-10). Oudere patiënten hebben meer medicijngebruik, comorbiditeiten, sociale behoefte en zijn kwetsbaarder door hun cognitieve status $(3,8-11)$. Ze hebben, vergeleken met jongeren, vaak een ernstiger ziekte presentatie, krijgen meer diagnostische testen, hebben een langere bezoektijd op de SEH en een verhoogd risico op een herbezoek aan de SEH $(3,4,12)$. Daarnaast hebben ze een hoger risico op ziekenhuisopname en overlijden ten opzichte van jongvolwassenen $(3,4,12)$. Factoren die bijdragen aan deze kwetsbaarheid zijn comorbiditeiten, hoge leeftijd, gebrek aan sociale steun, polyfarmacie, verminderd cognitief functioneren en depressie $(3,13)$.

Zorgverleners op de SEH zien de oudere patiënten als een uitdagende groep (14). Over het algemeen hebben internisten en geriaters de meeste ervaring met deze patiëntengroep (15). Het is echter bewezen dat zorgverleners nog steeds te weinig aandacht hebben voor deze kwetsbare groep (3). Dit komt voornamelijk door het gebrek aan ervaring / training (3). Daarnaast is het diagnostisch proces vaak tijdrovend en is het lastig om accurate informatie te verkrijgen. Deze tijd en bronnen zijn echter schaars op een drukke $\mathrm{SEH}$. Daarom is het essentieel om meer inzicht te krijgen in deze oudere patiëntengroep op de SEH.

Een specifieke groep met een specifieke zorgbehoefte is de oudere patiënten met niet specifieke klachten (16-21). Patiënten met niet specifieke klachten hebben voornamelijk algehele malaise, zonder specifieke klachten die snel wijzen op een bepaalde diagnose, zoals pijn op de borst of kortademigheid.

Het doel van dit proefschrift was dan ook om inzicht te verkrijgen in de potentiële risicofactoren, zoals weekend opname en sociaal economische status, voor oudere patiënten op de SEH. Deze risicofactoren kunnen mogelijk leiden tot het ontwikkelen van succesvolle interventies welke bijdragen aan de verbetering van de zorg en de uitkomsten voor deze oudere patiënten op de SEH. Daarnaast heeft het proefschrift als doel om meer inzicht te krijgen in oudere patiënten die zich presenteren met niet 
specifieke klachten op de SEH. Dit hebben we gedaan door het opzetten van enkele retrospectieve onderzoeken in het Máxima Medisch Centrum, te Veldhoven.

Het proefschrift is opgedeeld in vier onderdelen: het eerste gedeelte is gericht op het creëren van een overzicht van de huidige acute interne zorg in Nederland. Het tweede hoofdstuk focust op de risicofactoren (zoals weekend opname en de sociaal economische status) en de gezondheidsuitkomsten van oudere patiënten op de SEH. Het volgende hoofdstuk richt zich op de uitkomsten van de oudere patiënten met niet specifieke klachten op de SEH. Het laatste hoofdstuk richt zich op de haalbaarheid van telemonitoring voor oudere patiënten met niet specifieke klachten in de thuissituatie.

In Hoofdstuk 2 wordt de organisatie van de Nederlandse acute zorg in kaart gebracht. De organisatie van de acute zorg heeft invloed op de prestatie van de SEH. In Nederland, wisselt deze organisatie per ziekenhuis. Tot op het moment was er geen specifieke informatie bekend over de details van deze organisatie. Een overzicht van de huidige situatie kan bijdragen aan het reorganiseren van de SEH zorg in Nederland. Hoofdstuk $\mathbf{2}$ geeft een overzicht van de organisatiestructuur en de verschillende rollen en verantwoordelijkheden van internisten en SEH-artsen door middel van een observationele studie. In de studie beantwoordden 76 ziekenhuizen vragen over de structuur van de SEH. We vonden dat er verschillen in de aanwezigheid van internisten en SEH-artsen, en ook verschillen in de werkafspraken.

Daarnaast onderzochten we het aantal SEH bezoeken van patiënten op de SEH. Het aantal patiënten daalde tussen januari 2013 en december 2017. Het aantal oudere patiënten steeg echter in deze tijdsperiode. Deze stijging van oudere patiënten op de SEH benadrukt het belang van een goed georganiseerde acute zorg daar zij vaak meer complexe zorgvragen hebben.

Het tweede dee/ van het proefschrift richt zich op de risicofactoren van oudere patiënten op de SEH in het Máxima Medisch centrum, te Veldhoven. Hoofdstuk 3 onderzoekt het risico op slechtere uitkomst bij een weekend opname ten opzichte van een week opname. In meerdere studies is aangetoond dat een bezoek gedurende het weekend op de SEH een hoger risico geeft op mortaliteit ten opzichte van een week bezoek. Tot op heden was nog niet onderzocht of oudere interne geneeskunde patiënten op de SEH ook een verhoogd risico hebben op overlijden.

In hoofdstuk 3 werd door middel van een retrospectief onderzoek gekeken naar het risico op overlijden binnen 2 en 30 dagen na het SEH bezoek. We vonden geen verschil in 2 en 30 dagen mortaliteit tussen weekend en weekdag opname. Opvallend was echter dat patiënten gedurende het weekend een hogere comorbiditeit en urgentie niveau hadden ten opzichte van patiënten die waren opgenomen gedurende de weekdagen. 
Deze resultaten pleiten ervoor dat de organisatie die gedurende het weekend verschilt van die tijdens kantooruren geen invloed heeft op de kwaliteit van zorg.

Tot op heden was het onbekend of het weekend effect bijdraagt aan een hoger risico op slechte uitkomsten voor oudere patiënten. De ziekenhuismortaliteit bij oudere patiënten opgenomen in het weekend was in onze studie $11,4 \%$, welke in vergelijking met andere studies fors hoger is (4,5-5,2\%) (22-26). Deze studies zijn vaak wel gebaseerd op een ziekte presentatie in alle leeftijdscategorieën, waarin onze studie populatie alleen oudere patiënten betrof. Hierdoor wordt ook een hoger overlijdenspercentage verwacht. Het weekend effect dat gevonden is in andere studies kan voornamelijk verklaard worden door de hogere kans op comorbiditeiten, en vanwege het feit dat gedurende het weekend een overplaatsing naar bijvoorbeeld een hospice minder makkelijk geregeld kan worden. Een mogelijke oorzaak zou kunnen zijn dat huisartsen adequaat en tijdig verwijzen gedurende de week en in het weekend.

In hoofdstuk $\mathbf{4}$ gaat in op het risico op opname en overlijden met een verschillende sociaal economische status (SES). We onderzochten in een groot retrospectieve studie de associatie tussen SES met een SEH bezoek. We vonden dat oudere thuiswonende patiënten met een lage SES een hoger risico hebben op een ziekenhuisopname dan patiënten met een hoge SES. Daarnaast hebben patiënten met een lage SES vaker een hogere triage categorie, ondergaan ze meer diagnostische onderzoeken en hebben ze een langer SEH verblijfsduur in vergelijking met hogere SES groepen. De ziekenhuismortaliteit en herbezoeken aan de SEH waren echter niet verschillend tussen de SES groepen. Dit is in contrast met enkele andere studies waar wel een hogere ziekenhuis mortaliteit en heropname risico werd gevonden voor patiënten met een lage SES.

Onze studie laat zien dat in een land met een relatief gelijke toegang tot zorg, ongeacht de SES, maar minimale verschillen zijn waargenomen tussen de verschillende SES groepen. Zorgverleners zullen zich echter bewust moeten zijn van de potentiële verschillen tussen de SES groepen.

Het derde gedeelte van het proefschrift onderzoekt de patiënten met niet specifieke klachten op de SEH. De definities van niet specifieke klachten verschillen echter per hoofdstuk. Hoofdstuk $\mathbf{5}$ is een retrospectieve studie met als conclusie dat oudere patiënten met niet specifieke klachten meer comorbiditeiten hebben en er is vaker sprake van polyfarmacie in vergelijking met patiënten met specifieke klachten. Daarnaast hebben oudere patiënten met niet specifieke klachten een hoger risico op ziekenhuisopname en overlijden dan patiënten met specifieke klachten.

We geven een gedetailleerd overzicht van de karakteristieken en de uitkomsten van oudere patiënten op de SEH die zich presenteren met niet specifieke klachten in vergelijking met specifieke klachten. De definitie van niet specifieke klachten in dit 
onderzoek was gebaseerd op de aanmeldklacht op de SEH na overdracht van de huisarts bij de verwijzing. De prevalentie van oudere niet specifieke klachten patiënten is $13,7 \%$. De patiënten met niet specifieke klachten worden vaker opgenomen en hebben een langere opnameduur. De meest belangrijke bevinding is dat patiënten een hoger risico hebben op overlijden binnen 30 dagen met niet specifieke klachten. De enige uitkomst die niet verschilde was het aantal herbezoeken SEH binnen 90 dagen.

We hebben aangetoond dat oudere patiënten met niet specifieke klachten meer comorbiditeiten hebben en meer medicijnen gebruiken. Deze factoren in combinatie met functionele problemen en communicatieproblemen kunnen mogelijk de ontwikkeling van niet specifieke klachten verklaren. Hierdoor wordt het diagnostische proces gecompliceerd op de SEH. Daarnaast zijn we waarschijnlijk vaak niet in staat om bij deze patiënten een diagnose te vinden aangezien ze werden geclassificeerd onder de groep 'not otherwise classified' (36.5\%) op het moment van ontslag van de SEH. Deze resultaten onderstrepen de ernst van presentatie en de complexiteit die dat meebrengt bij niet specifieke klachten.

Hoofdstuk 6 onderzocht alle patiënten (leeftijd > 18 jaar) met niet specifieke klachten door middel van een specifiek algoritme door bepaalde items die kunnen worden verkregen in het triageproces. Het algoritme van een andere studie, de BANC studie (16), werd gebruikt. De patiënten waren opnieuw meestal ouder, hadden meerdere comorbiditeiten en polyfarmacie dan patiënten met specifieke klachten. Deze resultaten komen overeen met de onderzochte patiëntenpopulatie van hoofdstuk $\mathbf{5}$. Daarentegen was het aantal geïncludeerde patiënten wel kleiner ten opzichte van de patiëntenpopulatie van hoofdstuk 5 (13.7\% vs. 4.2\%). De voornaamste oorzaak was waarschijnlijk de manier van includeren, waarbij in de eerste studie gebruikt werd gemaakt van de verwijsindicatie en in de tweede studie van de data zoals bekend van anamnese en lichamelijk onderzoek. We denken dat deze gedetailleerde informatie belangrijk is voor het stellen van de diagnose van niet specifieke klachten.

De patiënten met niet specifieke klachten hadden een langer verblijf op de SEH, werden vaker opgenomen, hadden een langer ziekenhuisverblijf en een substantieel hoger risico op ziekenhuis mortaliteit in vergelijking met patiënten met specifieke klachten. Ondanks het verschil in definitie tussen hoofdstuk $\mathbf{5}$ en $\mathbf{6}$ hadden beide studies dezelfde uitkomsten. Onze studie laat zien dat het mogelijk is om gebruik te maken van het algoritme gedurende het diagnostische traject op de SEH om patiënten met niet specifieke klachten te identificeren die een verhoogd risico hebben op slechte uitkomsten.

In hoofdstuk 7 werd onderzocht dat heropname van patiënten met niet specifieke klachten niet voorspelbaar of te voorkomen is. 
In een retrospectief onderzoek onderzochten we patiënten met niet specifieke klachten in en patiënten met specifieke klachten en het risico op heropname. Hiervoor werd gebruikt gemaakt van de CURIOSA studie (27), een prospectief observationeel onderzoek (1398 patiënten) naar het vermijdbaarheid van heropnames door interviews met patiënten, mantelzorgers, artsen en verpleegkundige. We vonden geen verschil tussen herbezoeken bij patiënten met niet specifieke klachten en patiënten met specifieke klachten tijdens initiële presentatie, behalve dat niet specifieke klachten patiënten meer comorbiditeiten hadden. Ongeveer $1 / 3$ van de patiënten vond dat hun heropname voorspelbaar was. De niet specifieke klachten patiënten vonden dat hun heropname minder vaak voorkomen had kunnen zijn vergeleken met de heropnames van patiënten met specifieke klachten (14.9\% vs. 25.4\%). De studie was echter initieel niet opgesteld om deze specifieke patiëntengroep te onderzoeken. Hierdoor is meer inzicht nodig in de patiënten visie van de heropnames tussen beide groepen.

Het laatste gedeelte focust op de eerste lijn (huisartsengeneeskunde). Idealiter willen we deze patiëntengroep met niet specifieke klachten reeds in de thuissituatie al beter in kaart brengen, en een mogelijkheid hiervoor zou telemonitoring kunnen zijn. Telemonitoring is het meten op afstand van vitale parameters (zoals ademhalingsfrequentie en polsfrequentie) door middel van ICT toepassingen. Hoofdstuk $\mathbf{8}$ onderzoekt de inzetbaarheid van telemonitoring voor oudere patiënten in de thuissituatie. We maakten gebruik van een draagbare sensor (van Philips) voor het registeren van vitale parameters (temperatuur, ademhalingsfrequentie, polsfrequentie, saturatie en bewegingen). We selecteerden 20 kwetsbare oudere patiënten met niet specifieke klachten in de thuissituatie (zoals de definitie van hoofdstuk 6). Daarnaast selecteerden we nog 10 controle patiënten zonder comorbiditeiten. We evalueerden (door middel van interviews) de patiënt ervaringen met een draagbare sensor in de thuissituatie.

We concludeerde dat telemonitoring in de thuissituatie mogelijk is m.b.t. de haalbaarheid en de tolerantie. Oudere patiënten hadden wel sturing nodig om de draagbare sensor te gebruiken tijdens de studie. Familieleden en de onderzoeker waren voor het grootste gedeelte nodig voor assistentie tijdens het onderzoek. Patiënten waren uiteindelijk wel enthousiast over de toepassing. De draagbare sensor gebruikt in de studie was op dat moment echter nog niet geschikt voor betrouwbare monitoring in verband met storingen van het apparaat bij problemen in de connectiviteit van het apparaat. De feedback kan worden gebruikt voor het opzetten van vervolgstudies met het gebruik van de draagbare sensor. 


\section{References}

1. Volkgezondheidszorg. https://www.volksgezondheidenzorg.info/onderwerp/bevolking.

2. Betty Meyboom-de Jong, Klaske Wynia and Anjo Geluk-Bleumink. Ageing better in the Netherlands. 2018.

3. Samaras N, Chevalley T, Samaras D, Gold G. Older patients in the emergency department: a review. Ann Emerg Med 2010 Sep;56(3):261-269.

4. Aminzadeh F, Dalziel WB. Older adults in the emergency department: a systematic review of patterns of use, adverse outcomes, and effectiveness of interventions. Ann Emerg Med 2002 Mar;39(3):238-247.

5. Marieke Buijs. overspoelen kwetsbare thuiswonende ouderen de spoedeisende hulp? Ned Tijdschr Geneeskd 2017:161.

6. Denman SJ, Ettinger WH, Zarkin BA, Coon PJ, Casani JA. Short-term outcomes of elderly patients discharged from an emergency department. J Am Geriatr Soc 1989 Oct;37(10):937-943.

7. Drame M, Jovenin N, Novella JL, Lang PO, Somme D, Laniece I, et al. Predicting early mortality among elderly patients hospitalised in medical wards via emergency department: the SAFES cohort study. J Nutr Health Aging 2008 Oct;12(8):599-604.

8. Hwang U, Morrison RS. The geriatric emergency department. J Am Geriatr Soc 2007 Nov;55(11):1873-1876.

9. Lowenstein SR, Crescenzi CA, Kern DC, Steel K. Care of the elderly in the emergency department. Ann Emerg Med 1986 May;15(5):528-535.

10. Salvi F, Morichi V, Grilli A, Giorgi R, De Tommaso G, Dessi-Fulgheri P. The elderly in the emergency department: a critical review of problems and solutions. Novermber 2007 Internal Emergency Medicine(2):292-292-301.

11. Schrijver EJ, Toppinga Q, de Vries OJ, Kramer MH, Nanayakkara PW. An observational cohort study on geriatric patient profile in an emergency department in the Netherlands. Neth J Med 2013 Jul-Aug;71(6):324-330.

12. Casalino E, Wargon M, Peroziello A, Choquet C, Leroy C, Beaune S, et al. Predictive factors for longer length of stay in an emergency department: a prospective multicentre study evaluating the impact of age, patient's clinical acuity and complexity, and care pathways. Emerg Med J 2013 Feb 28.

13. Bulut $H$, Yazici G, Demircan A, Keles A, Guler Demir S. Determining emergency physicians' and nurses' views concerning older patients: a mixed-method study. Int Emerg Nurs 2014 Aug 11.

14. Perry A, Macias Tejada J, Melady D. An Approach to the Older Patient in the Emergency Department. Clin Geriatr Med 2018 Aug;34(3):299-311.

15. Kremers MNT, Wachelder JJH, Nanayakkara PWB, Haak On Behalf Of The Orca Onderzoeks Consortium Acute Geneeskunde Acute Medicine Research Consortium,H.R. Organisation of internal medicine in acute care in the Netherlands: a detailed overview. Neth J Med 2020 Sep;78(5):251-260.

16. Nemec M, Koller MT, Nickel CH, Maile S, Winterhalder C, Karrer C, et al. Patients presenting to the emergency department with non-specific complaints: the Basel Non-specific Complaints (BANC) study. Acad Emerg Med 2010 Mar;17(3):284-292. 
17. Nickel $\mathrm{CH}$, Nemec $\mathrm{M}$, Bingisser $\mathrm{R}$. Weakness as presenting symptom in the emergency department. Swiss Med Wkly 2009 May 2;139(17-18):271-272.

18. Djarv T, Castren M, Martenson L, Kurland L. Decreased general condition in the emergency department: high in-hospital mortality and a broad range of discharge diagnoses. Eur J Emerg Med 2015 Aug;22(4):241-246.

19. Karakoumis J, Nickel CH, Kirsch M, Rohacek M, Geigy N, Muller B, et al. Emergency Presentations With Nonspecific Complaints-the Burden of Morbidity and the Spectrum of Underlying Disease: Nonspecific Complaints and Underlying Disease. Medicine (Baltimore) $2015 \mathrm{Jul} ; 94(26): \mathrm{e} 840$.

20. Kemp K, Mertanen R, Laaperi M, Niemi-Murola L, Lehtonen L, Castren M. Nonspecific complaints in the emergency department - a systematic review. Scand J Trauma Resusc Emerg Med 2020 Jan 28;28(1):6.

21. Safwenberg U, Terent A, Lind L. The Emergency Department presenting complaint as predictor of in-hospital fatality. Eur J Emerg Med 2007 Dec;14(6):324-331.

22. Aylin P, Yunus A, Bottle A, Majeed A, Bell D. Weekend mortality for emergency admissions. A large, multicentre study. Qual Saf Health Care 2010 Jun;19(3):213-217.

23. Bell CM, Redelmeier DA. Mortality among patients admitted to hospitals on weekends as compared with weekdays. N Engl J Med 2001 Aug 30;345(9):663-668.

24. Freemantle N, Richardson M, Wood J, Ray D, Khosla S, Shahian D, et al. Weekend hospitalization and additional risk of death: an analysis of inpatient data. J R Soc Med 2012 Feb;105(2):74-84.

25. Khanna R, Wachsberg K, Marouni A, Feinglass J, Williams MV, Wayne DB. The association between night or weekend admission and hospitalization-relevant patient outcomes. J Hosp Med 2011 Jan;6(1):10-14.

26. Lapointe-Shaw L, Bell CM. It's not you, it's me: time to narrow the gap in the weekend care. BMJ Qual Saf 2013;0:1-3.

27. L. van Galen. Patient safety in the acute healthcare chain; is it safer@home? 2017. 